\title{
DYNAMICS ANALYSIS AND PLANNING FOR A SPECIFIC LEG MODEL WITH A VARIABLE STIFFNESS ELEMENT
}

\author{
Li, Z.; Tan, Y. G.; Zeng, S. \& Luo, H. Y. \\ School of Mechanical and Electronic Engineering, Wuhan University of Technology, Wuhan 430070, \\ Hubei Province, China \\ E-Mail: 1z2015@whut.edu.cn,ygtan@whut.edu.cn
}

\begin{abstract}
Variable compliant elements in the leg play an important role in quadruped mammals' locomotion. This paper mainly investigates the dynamics characteristics of a specific leg model with a variable stiffness element during the stance phase. By choosing appropriate initial states and planning joint motion under specific constraint conditions, the model is able to move normally and pivot about the contact point. Particularly, the passive motion of the variable stiffness element is planned based on forced vibration characteristics and is set to experience a compression and extension process. In a detailed study, the optimal combination of the system parameters, meeting constraint conditions, were evaluated. We investigated the effects of the initial states on the leg's motion, and obtained the necessary variable stiffness characteristics under different conditions. The ground reaction features of the leg model present similar characteristics to those of a canine. A variable stiffness device with a good adjustability feature and a large adjustable range is discussed.

(Received, processed and accepted by the Chinese Representative Office.)
\end{abstract}

Key Words: Leg Model, Variable Stiffness, Quadruped Robots, Dynamics Analysis, Motion Creation

\section{INTRODUCTION}

The leg is the most important component for quadruped mammals' locomotion. When quadruped mammal moves, its compliant elements play important roles in saving energy and moderating the impact with the ground [1]. The compliant elements, such as muscles, tendons and ligaments, are able to facilitate the energy transformation during the stance phase [2]. Especially, under the compliant effects, the efficiency in running can reach up to $50 \%$ [3]. Inspired by the compliant effects of quadruped running, compliant elements were added into legs to improve the performance of quadruped robots [4]. These robots are successful, when the stiffness of their compliant elements are fixed, which is not enough when robots need to face a more complex environment. When the locomotion has highly dynamic effects, the oscillation feature of a fixed stiffness spring in the system will lead to uncertain movement and instability that cannot be controlled easily. It has been shown that human and animal runners can adjust their leg stiffness allowing them to maintain similar running mechanics when the interaction conditions between the foot and ground change [5]. Therefore, the variable compliant property will play an important role in this situation [6].

To account for the dynamic process of legged running, the Spring Loaded Inverted Pendulum (SLIP) was modelled [7], which reflects the intuitive motion mechanism in terms of biomechanics. This model assumes that the leg locomotion can be described as a massless linear spring leg connected to a point mass body. The mass pivots about the contact point, and the spring experiences periodic compression and extension. Despite the model's simplicity, it accurately reflects the interactive characteristic with the ground and the body motion. Based on the SLIP model, many models were developed to further study quadruped motion, such as the two-dimensional bounding model $[8,9]$. The common feature in these models is that the leg is simplified as a linear spring, and the detailed motion of the leg structure is not taken into account. In view of that the complexity of the model is one of the important factors 
affecting the simulation result for real mechanisms; the motion has been demonstrated to result from the coordination of every component, including muscles, tendons and ligaments [10]. To study the detailed motion of the leg structure, a bio-inspired model with respect to specific robot leg should be established and studied. In this paper, the dynamic equations of the model that are sensitive to variable stiffness were derived and simulated using numerical methods, as opposed to the method of using the co-simulation of MATLAB and ADAMS to establish the model [11].

In the previous work [12], we proposed a specific single-leg model. This single-leg model has a similar structural configuration with some current quadruped robot legs and contains a variable stiffness element. In the dynamic analysis, the generalized form of the dynamics equation was derived. The generalized parameter of the leg motion in the dynamics equations was chosen to study the dynamic characteristics of the specific leg model and its sensitivity to the variable stiffness. In this paper, we evaluated the optimal combination of these generalized parameters under certain motion constraint conditions. By varying the kinematics parameters, the dynamic characteristics and the required variable stiffness were analysed. The dynamics analysis was carried out during the stance phase because the variable stiffness mainly influences the interaction characteristics of the leg with the ground. The possible implementation of a device is also discussed.

The paper is organized as follows. Section 2 gives a simple introduction for this leg model and dynamics analysis. Section 3 illustrates the method of motion creation and the simulation experiment for choosing parameters. The dynamics simulation results of the leg model are shown and discussed in Section 4. Conclusions and implications for future work are given in Section 5 .

\section{SINGLE LEG MODEL}

\subsection{Structural configuration}

The leg model proposed in the previous work [12] consists of the following three segments from top to bottom: thigh, shank and foot. Motor 1 located in the rack drives the thigh to swing. Complying with the design principle of reducing the inertia of the leg [13], a link mechanism connecting the rack and the shank is used to transmit the torque of motor 2 and drive the shank. Consequently, motor 1 and motor 2 are both located in the rack. To drive the foot segment, some used a parallel four-bar mechanism to synchronize the foot with thigh [14], and some used a linear spring to make the ankle joint compliant [15]. In this paper, a variable stiffness element is applied to replace the link and act as the gastrocnemius muscle in mammals. Then, the compliant ankle joint is able to be regulated to create different dynamic motions.

This motion belongs to the multi-body dynamics problem, and all motions are restricted to the sagittal plane. It is assumed that the rack cannot rotate to imitate the horizontal body of quadruped robots and is simplified as a point mass $m$ connected to the hip joint. Its position coordinate in the inertial frame is $(x, z)$. The parameters of the leg model are listed in Table I, where $\left(m_{i}, l_{i}, J_{i}\right)(i=1,2,3)$ indicate the weight, length and inertia of the thigh, shank and foot segments, respectively. $\left(l_{s 1}, l_{s 2}, l_{s 3}\right)$ indicate the locations of the centre of mass of each segment. It is assumed that the links of the four-bar mechanism are massless to simplify the multi-body dynamics. The variable stiffness spring is also regarded as massless. 


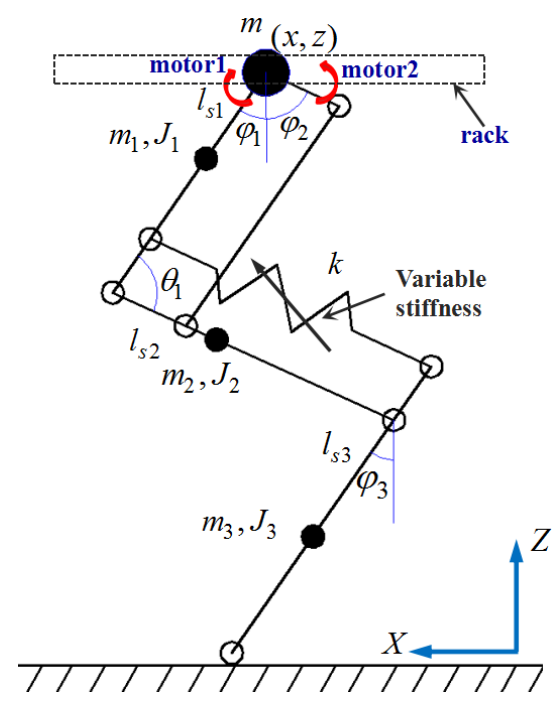

Figure 1: The single leg model.

Table I: List of model parameters and values.

\begin{tabular}{|l|c|c|c|}
\hline \multicolumn{1}{|c|}{ Part } & Weight $[\mathrm{kg}]$ & Length $[\mathrm{m}]$ & Barycentre location $[\mathrm{m}]$ \\
\hline Rack & 2.2 & & \\
\hline Thigh & 0.165 & 0.145 & 0.04 \\
\hline Shank & 0.4 & 0.17 & 0.09 \\
\hline Foot & 0.078 & 0.107 & 0.035 \\
\hline
\end{tabular}

\subsection{System dynamics}

The dynamics equations of leg motion are stated in the form as follows:

$$
\mathbf{M}(\mathbf{q}) \ddot{\mathbf{q}}+\mathbf{B}(\mathbf{q})[\dot{\mathbf{q}} \dot{\mathbf{q}}]+\mathbf{C}(\mathbf{q})\left[\dot{\mathbf{q}}^{2}\right]=\left(\begin{array}{c}
\tau_{s 1} \\
\tau_{s 2} \\
0
\end{array}\right)-\mathbf{G}_{q}\left(\begin{array}{c}
m_{1} g \\
m_{2} g \\
m_{3} g
\end{array}\right)-\mathbf{F}_{q}\left(\begin{array}{c}
F_{f} \\
F_{n}
\end{array}\right)-\mathbf{M}_{s q} k l_{30}^{2}\left(\varphi_{1}-\varphi_{3}\right)
$$

where $\mathbf{M}(\mathbf{q})$ is the mass matrix and is given as:

with

$$
\mathbf{M}(\mathbf{q})=\left[\begin{array}{cccc}
2 J_{00} & J_{10} & J_{20} & J_{30} \\
J_{10} & 2 J_{11} & J_{12} & J_{13} \\
J_{20} & J_{12} & 2 J_{22} & J_{23} \\
J_{30} & J_{13} & J_{23} & 2 J_{33}
\end{array}\right]
$$

$$
\begin{aligned}
& J_{00}=J_{11}+J_{22}+A_{1} \cos \left(\varphi_{1}+\varphi_{2}\right), \quad J_{11}=\frac{1}{2}\left(J_{1}+m l_{1}^{2}+m_{1}\left(l_{1}-l_{s 1}\right)^{2}\right), \quad J_{22}=\frac{1}{2}\left(J_{2}+m l_{2}^{2}+m_{1} l_{2}^{2}+m_{2}\left(l_{2}-l_{s 2}\right)^{2}\right), \\
& J_{33}=\frac{1}{2}\left(J_{3}+m l_{3}^{2}+m_{1} l_{3}^{2}+m_{2} l_{3}^{2}+m_{3}\left(l_{3}-l_{s 3}\right)^{2}\right), \quad J_{12}=-A_{1} \cos \left(\varphi_{1}+\varphi_{2}\right), \quad J_{10}=2 J_{11}+A_{1} \cos \left(\varphi_{1}+\varphi_{2}\right), \\
& J_{20}=-2 J_{22}-A_{1} \cos \left(\varphi_{1}+\varphi_{2}\right), \quad J_{13}=A_{3} \cos \left(\varphi_{1}-\varphi_{3}\right), \quad J_{23}=-A_{2} \cos \left(\varphi_{2}+\varphi_{3}\right), \quad J_{30}=A_{2} \cos \left(\varphi_{2}+\varphi_{3}\right)+A_{3} \cos \left(\varphi_{1}-\varphi_{3}\right), \\
& A_{1}=m l_{1} l_{2}+m_{1} l_{2}\left(l_{1}-l_{s 1}\right), \quad A_{2}=m l_{2} l_{3}+m_{1} l_{2} l_{3}+m_{2} l_{3}\left(l_{2}-l_{s 2}\right), \quad A_{3}=m l_{1} l_{3}+m_{1} l_{3}\left(l_{1}-l_{s 1}\right)
\end{aligned}
$$

$\mathbf{B}(\mathbf{q})$ is the Coriolis matrix and $\mathbf{B}(\mathbf{q})[\dot{\mathbf{q}} \dot{\mathbf{q}}]=0 . \mathbf{C}(\mathbf{q})$ is the centrifugal matrix and is given as:

$$
\mathbf{C}(\mathbf{q})=\left[\begin{array}{cccc}
0 & -A_{1} \sin \left(\varphi_{1}+\varphi_{2}\right) & A_{1} \sin \left(\varphi_{1}+\varphi_{2}\right) & -A_{2} \sin \left(\varphi_{2}+\varphi_{3}\right)+A_{3} \sin \left(\varphi_{1}-\varphi_{3}\right) \\
A_{1} \sin \left(\varphi_{1}+\varphi_{2}\right) & 0 & A_{1} \sin \left(\varphi_{1}+\varphi_{2}\right) & A_{3} \sin \left(\varphi_{1}-\varphi_{3}\right) \\
A_{1} \sin \left(\varphi_{1}+\varphi_{2}\right) & A_{1} \sin \left(\varphi_{1}+\varphi_{2}\right) & 0 & A_{2} \sin \left(\varphi_{2}+\varphi_{3}\right) \\
A_{2} \sin \left(\varphi_{2}+\varphi_{3}\right)-A_{3} \sin \left(\varphi_{1}-\varphi_{3}\right) & -A_{3} \sin \left(\varphi_{1}-\varphi_{3}\right) & A_{2} \sin \left(\varphi_{2}+\varphi_{3}\right) & 0
\end{array}\right]
$$

$\mathbf{q}=\left[\varphi_{1}, \varphi_{2}, \varphi_{3}\right]^{\mathrm{T}}$ indicates the generalized coordinate vector of the model, which are the elevation angles of each segment relative to the vertical direction. The $\varphi_{1}$ and $\varphi_{2}$ also refer to 
the driven angles of motor 1 and motor 2, respectively. The items on the right side of Eq. (1) indicate the vector of generalized torque mapped on the generalized coordinates, including driven torque $\left[\tau_{s 1}, \tau_{s 2}\right]^{\mathrm{T}}$ and the torque mapped by gravity, ground reaction force and elastic force. $-\mathbf{G}_{q},-\mathbf{F}_{q}$ and $-\mathbf{M}_{s q}$ respectively indicate the coefficient matrix of generalized torque of gravity, ground reaction force and elastic force. These coefficient matrices are given respectively as:

$$
\begin{gathered}
\mathbf{G}_{q}=\left[\begin{array}{ccc}
l_{s 1} \sin \varphi_{1} & l_{1} \sin \varphi_{1} & l_{1} \sin \varphi_{1} \\
0 & l_{s 2} \sin \varphi_{2} & l_{2} \sin \varphi_{2}-l_{s 3} \sin \varphi_{3} \\
0 & 0 & l_{s 3} \sin \varphi_{3}
\end{array}\right] \\
\mathbf{F}_{q}=\left[\begin{array}{cc}
-l_{1} \cos \varphi_{1} & -l_{1} \sin \varphi_{1} \\
l_{2} \cos \varphi_{2}+l_{3} \cos \varphi_{3} & -l_{2} \sin \varphi_{2}+l_{3} \sin \varphi_{3} \\
-l_{3} \cos \varphi_{3} & -l_{3} \sin \varphi_{3}
\end{array}\right] \\
\mathbf{M}_{s q}=\left[\begin{array}{c}
\sin \left(\varphi_{1}+\varphi_{2}\right) \\
\sin \left(\varphi_{1}+\varphi_{2}\right) \\
-\sin \left(\varphi_{1}+\varphi_{2}\right)
\end{array}\right]
\end{gathered}
$$

$F_{f}$ and $F_{n}$ refer to the ground reaction force in the horizontal and vertical directions, respectively, and are set to meet the centroid theorem. $k$ is the variable stiffness of the compliant element, which is an independent value, and $l_{30}$ is the length between the knee joint and the point where a spring connects to the thigh. Because the deflection of the ankle joint is set small, the elastic torque on the ankle is expressed as $\tau=k l_{30}\left(\varphi_{3}-\varphi_{1}\right)$.

The driven torque has effects on the motion dynamics, and the system is sensitive to the variable stiffness. It is worth noting that there is no driven torque in the third row of Eq. (1). This means that the motion of the ankle joint decouples with driven torque, but couples with the motion of the other two joints. To eliminate the influences of driven torque in the analysis, the dynamics equations are partitioned into two parts and the third row of the equations is extracted. Then, the equation without driven torque can be obtained and it reflects the coupling relationship between joint motion and stiffness.

The knee joint motion $\theta_{1}$ results from the coupling of $\varphi_{1}$ and $\varphi_{2}, \theta_{1}=\pi-\varphi_{1}-\varphi_{2}$. The deflection of the ankle joint can be expressed as $\varphi=\varphi_{3}-\varphi_{1}$. Substituting the deflection $\varphi$ of the ankle joint into the partitioned equation, the motion of the foot segment $\varphi_{3}$ is replaced by $\varphi$, and the coupling dynamics equation of $\varphi$ and $\left(\varphi_{1}, \varphi_{2}, k\right)$ is obtained as follows:

$$
\left\{\begin{array}{l}
M_{v} \ddot{\varphi}+K_{v} \varphi+C_{v}=0 \\
K_{v}=K_{v}\left(k, \varphi_{1}, \varphi_{2}, \dot{\varphi}_{1}, \dot{\varphi}_{2}, \ddot{\varphi}_{2}\right) \\
C_{v}=C_{v}\left(\varphi_{1}, \varphi_{2}, \dot{\varphi}_{2}, \ddot{\varphi}_{1}, \ddot{\varphi}_{2}\right)
\end{array}\right.
$$

\subsection{Generalized form}

The structure of Eq. (7) is similar to the dynamics formula for forced vibration. $\varphi$ is the elastic variable. $M_{v}$ is generalized mass. $K_{v}$ indicates the generalized stiffness. The generalized stiffness is influenced by system dynamic effects, which reflects generalized natural vibration characteristics. $C_{v}$ is regarded as the generalized vibration exciter, which is related to the kinematics of segments $\left(\varphi_{1}, \varphi_{2}\right)$. That means system dynamics effects also motivate the vibration of the passive ankle joint. Because of the lack of consideration of the damping in the complaint variable, there is no damping term in Eq. (7). Consequently, the deflection $\varphi$ of ankle joint is a superposed motion of forced response and free accompanying. By utilizing the features of forced vibration, Eq. (7) is written in the following form: 


$$
\left\{\begin{array}{l}
C_{v}\left(\varphi_{1}, \varphi_{2}, \dot{\varphi}_{2}, \ddot{\varphi}_{1}, \ddot{\varphi}_{2}\right)+\varphi_{m}\left(K_{v}-M_{v} \omega^{2}\right) \sin \omega t=0 \\
K_{v}\left(k, \varphi_{1}, \varphi_{2}, \dot{\varphi}_{1}, \dot{\varphi}_{2}, \ddot{\varphi}_{2}\right)-\frac{\pi^{2} M_{v}}{T_{d}^{2} s^{2}}=0
\end{array}\right.
$$

where $M_{v}$ is only related to structure parameters $\left(l_{i}, l, m_{i}, m\right)(i=1,2,3) . \varphi_{m}$ indicates the vibration amplitude caused by the generalized vibration exciter, and the amplitude of the generalized vibration exciter can be expressed as $\varphi_{m}\left(K_{v}-M_{v} \omega^{2}\right) . T_{d}$ is the excitation cycle and $\omega=\pi / T_{d}$ indicates the excitation frequency. $s$ is the ratio of excitation frequency to generalized inherent frequency and be can expressed as $s=\pi \sqrt{M_{v}} /\left(T_{d} \sqrt{K_{v}}\right)$. The controlled system parameters, excepting the kinematics parameters and $k$, include $T_{d}, s$ and $\varphi_{m}$. The motion of deflection $\varphi$ of the ankle joint is characterized as follows:

$$
\varphi(t)=-\frac{F_{0}}{K_{v}} \frac{s}{1-s^{2}} \sin \omega_{0} t+\frac{F_{0}}{K_{v}} \frac{1}{1-s^{2}} \sin \omega t
$$

\section{MOTION CREATION AND PARAMETERS}

\subsection{Motion constraints}

Because this paper focuses on the dynamics analysis of the leg during the stance phase, it is not a periodic problem. Therefore, certain kinetic conditions are used to restrain the leg system's locomotion to create a normal body-moving trajectory. The constraint conditions are shown in the following inequalities.

$$
\left\{\begin{array}{l}
\min (\dot{x})>0 \\
\mid z_{m}(\text { lift })-z_{m}(\text { stand }) \mid<0.01 m \\
\min \left(F_{n}\right)>0 \\
\max \left(\frac{F_{f}}{F_{n}}\right)<\mu_{s}
\end{array}\right.
$$

The constraint conditions mean that the motion must meet the following requirements: 1) the body mass must moves forward, 2) when the foot stands on the ground and is lifted off, the body mass height $z_{m}$ must be close which is beneficial for entering the swing phase and the next stance phase, 3) the foot must keep touching the ground to support the leg, 4) there is no slide between the foot and the ground which has a setting friction coefficient $\mu_{s}$. In the other words, the ground reaction friction meets the frictional angle.

Apart from these conditions, the deflection $\varphi$ of the ankle joint at lift-off time must be zero, meaning the spring returns to its original length. In the terms of Eq. (9), the deflection $\varphi$ is a superposed resulting motion. Therefore, the time parameter $t_{e}$ is defined to indicate stance duration. The vibration amplitude $\varphi_{m}$ is set to $20^{\circ}$.

Eq. (8) can be simplified into three first-order equations. However, there are five variables $\left(k, \varphi_{1}, \varphi_{2}, \dot{\varphi}_{1}, \dot{\varphi}_{2}\right)$, which cannot be solved by numerical computation. If the motion of segments $\left(\varphi_{1}, \varphi_{2}\right)$ is defined, the numerical computation can be carried out. The choice of $\varphi_{2}$ contributes to simplifying the computation and we chose $\varphi_{2}$ to plan around. To imitate the motion closer to quadruped runners, we captured dog motion in the sagittal plane using a high-speed camera (FASTCAM Mini UX100) at $250 \mathrm{~Hz}$ to obtain the kinematics characteristic of the shank segment $\varphi_{2}$. Through a large number of simulations, we fit the following $\varphi_{2}$ (unit: deg) that can produce more leg locomotion satisfying the above constraint conditions.

$$
\varphi_{2}(t)=2124.4 t^{3}-2082.2 t^{2}+503.1346 t+18.6
$$

Then, Eq. (8) can be simplified into three first-order equations with three variables $\left(k, \varphi_{1}, \dot{\varphi}_{1}\right)$. The dynamics computation is solved numerically with MATLAB using a 
Runge-Kutta iteration (ode45) algorithm. As a result, the parameters influencing dynamics characteristics include variable stiffness $(k)$, generalized vibration parameters $\left(T_{d}, s, t_{e}\right)$ and initial kinematic states $\left(\varphi_{10}, \dot{\varphi}_{10}\right)$.

\subsection{Generalized vibration parameters}

The $T_{d}$ determines the frequency of forced response, and $s$ indirectly determines the frequency of the free accompanying motion. As described in the previous work regarding the resulting superposed motion with $s$ changing [12] and the motion under the given conditions of the excitation cycle $\left(T_{d}=0.15\right)$, stance duration $\left(t_{e}=0.21\right)$, and initial kinematic states $\left(\varphi_{10}=37^{\circ}\right.$, $\dot{\varphi}_{10}=0.2 \mathrm{rad} / \mathrm{s}$ ) shown in Fig. 2, it is found that along with $s$ varying, the cycle of free accompanying motion changes, which results in the varying of superposed angular motion at the lift-off event. Therefore, the stance duration $t_{e}$ when the deflection $\varphi$ is exactly equal to zero results from the suitable choice of $T_{d}$ and $s$. Therefore, by computing the resulting characteristic of deflection $\varphi$, the optimum combination of generalized vibration parameters $\left(T_{d}, s, t_{e}\right)$ is obtained, as shown in Fig. 3. Accordingly, the combination of generalized stiffness $K_{v}=\pi^{2} M_{v} / T_{d}^{2} s^{2}$ with $T_{d}$ and $t_{e}$ is obtained (see Table II).

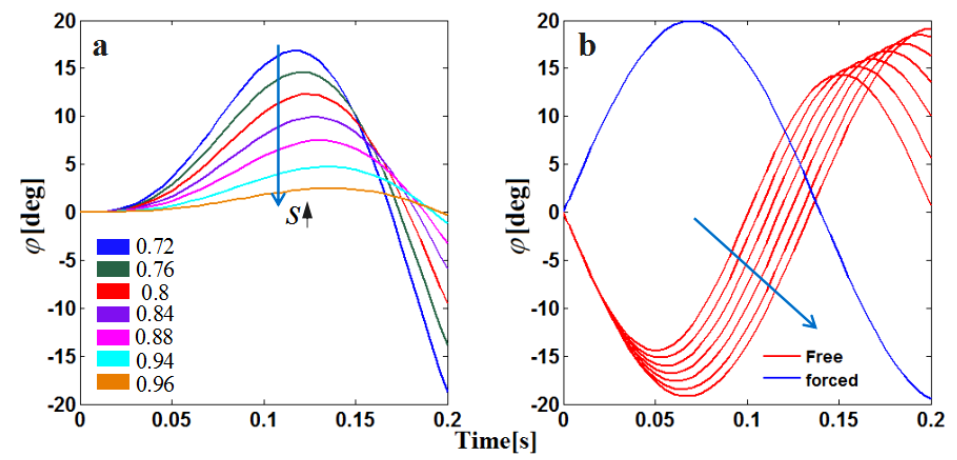

Figure 2: The deflection $\varphi$ of the ankle joint.

Table II: The list of combination of generalized stiffness $K_{v}\left(\pi^{2} M_{v} / T_{d}^{2} s^{2}\right)$ with $T_{d}$ and $t_{e}$.

\begin{tabular}{|c|c|c|c|c|c|c|c|}
\hline \multirow{2}{*}{$T_{d}[\mathrm{~s}]$} & \multicolumn{7}{|c|}{$K[\mathrm{~N} / \mathrm{m}]$} \\
\cline { 2 - 8 } & \multicolumn{3}{|c|}{ Time difference of stance duration and excitation cycle $t_{1}[\mathrm{~s}], t_{e}=T_{d}+t_{1}$} \\
\cline { 2 - 8 } & 0.000 & 0.010 & 0.020 & 0.030 & 0.040 & 0.050 & 0.060 \\
\hline 0.140 & 129.986 & 100.725 & 78.843 & 62.861 & 50.776 & 41.868 & 36.236 \\
0.150 & 113.232 & 87.743 & 68.681 & 54.758 & 44.231 & 36.472 & 31.565 \\
0.160 & 99.520 & 77.118 & 60.365 & 48.128 & 38.875 & 32.055 & 27.743 \\
0.170 & 88.156 & 68.312 & 53.472 & 42.632 & 34.436 & 28.395 & 24.575 \\
0.180 & 78.633 & 60.933 & 47.695 & 38.027 & 30.716 & 25.328 & 21.920 \\
0.190 & 70.574 & 54.687 & 42.807 & 34.129 & 27.568 & 22.732 & 19.674 \\
0.200 & 63.693 & 49.355 & 38.633 & 30.802 & 24.880 & 20.515 & 17.755 \\
0.210 & 57.771 & 44.767 & 35.042 & 27.938 & 22.567 & 18.608 & 16.105 \\
0.220 & 52.639 & 40.790 & 31.928 & 25.456 & 20.562 & 16.955 & 14.674 \\
0.230 & 48.161 & 37.320 & 29.212 & 23.290 & 18.813 & 15.513 & 13.426 \\
0.240 & 44.231 & 34.275 & 26.829 & 21.390 & 17.278 & 14.247 & 12.330 \\
0.250 & 40.764 & 31.587 & 24.725 & 19.713 & 15.923 & 13.130 & 11.363 \\
0.260 & 37.688 & 29.204 & 22.860 & 18.226 & 14.722 & 12.139 & 10.506 \\
0.270 & 34.948 & 27.081 & 21.198 & 16.901 & 13.652 & 11.257 & 9.742 \\
0.280 & 32.496 & 25.181 & 19.711 & 15.715 & 12.694 & 10.467 & 9.059 \\
\hline
\end{tabular}


Consequently, when the excitation cycle $T_{d}$ is fixed, the frequency ratio $s$ increases with stance duration $t_{e}$ increasing; on the contrary, the generalized stiffness $K_{v}$ decreases. In other words, the generalized frequency $\omega_{0}$ decreases. When the stance duration $t_{e}$ is fixed, the frequency ratio $s$ decreases with increasing excitation cycle $T_{d}$; on the contrary, the generalized stiffness $K_{v}$ increases. This means that when the stance duration $t_{e}$ is fixed, generalized frequency $\omega_{0}$ increases as excitation cycle $T_{d}$ increases. These are the results of superposed motion. It is worth noting that the superposed motion is accomplished without exceeding one cycle period because with an increasing number of vibrations in the cycle, a higher frequency motion is needed, and it becomes more difficult to keep the motion stable.

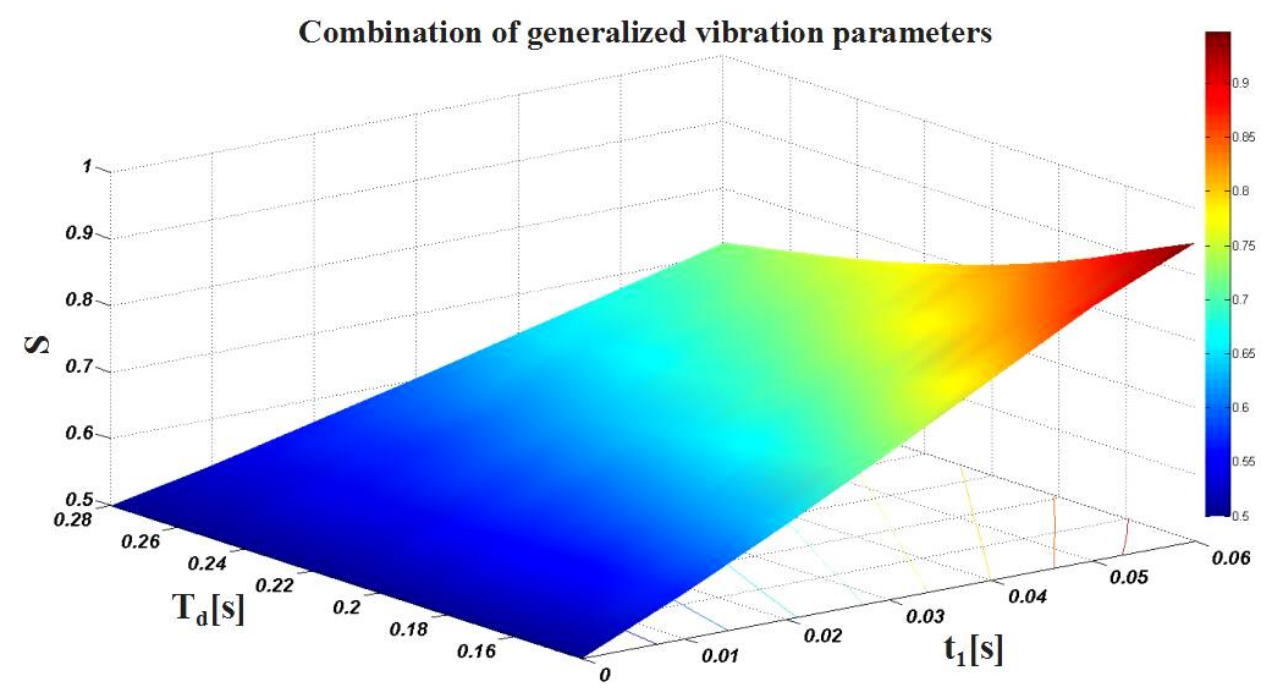

Figure 3: The optimum combination of generalized vibration parameters $\left(t_{1}=t_{e}-T_{d}\right)$.

Based on the combination of generalized vibration parameters, we investigated the normal motion meeting the constraint conditions of Eq. (10). In the simulation, it is obviously found that there are more suitable motion with a bigger friction coefficient: if $\mu_{s}$ is equal to 0.5 , there is satisfying motion only when $T_{d}$ is equal to $0.14 \mathrm{~s}$; if $\mu_{s}$ is equal to 0.6 , there is satisfying motion when $T_{d}$ is less than $0.16 \mathrm{~s}$; if $\mu_{s}$ is equal to 0.7 , there is satisfying motion when $T_{d}$ is less than $0.17 \mathrm{~s}$; if $\mu_{s}$ is equal to 0.8 , there is satisfying motion when $T_{d}$ is less than $0.19 \mathrm{~s}$.

Table III: List of range of initial values meeting constraint conditions when $\mu_{s}=0.6$.

\begin{tabular}{|c|c|c|c|c|}
\hline$T_{d}[\mathrm{~s}]$ & $t_{e}[\mathrm{~s}]$ & $s$ & $\varphi_{10}[\mathrm{deg}]$ & $\dot{\varphi}_{10}[\mathrm{rad} / \mathrm{s}]$ \\
\hline \multirow{11}{*}{0.14} & \multirow{11}{*}{0.2} & \multirow{11}{*}{0.947} & 37 & $-0.39 \sim 0.06$ \\
\hline & & & 38 & $-0.46 \sim 0.22$ \\
\hline & & & 39 & $-0.54 \sim 0.3$ \\
\hline & & & 40 & $-0.62 \sim 0.36$ \\
\hline & & & 41 & $-0.7 \sim 0.39$ \\
\hline & & & 42 & $-0.78 \sim 0.41$ \\
\hline & & & 43 & $-0.86 \sim 0.42$ \\
\hline & & & 44 & $-0.76 \sim 0.39$ \\
\hline & & & 45 & $-0.58 \sim 0.12$ \\
\hline & & & 46 & $-0.48 \sim 0.15$ \\
\hline & & & 47 & $-0.42 \sim 0.4$ \\
\hline \multirow{2}{*}{0.15} & \multirow{2}{*}{0.21} & \multirow{2}{*}{0.924} & 37 & $0.14 \sim 0.23$ \\
\hline & & & 38 & $0.06 \sim 0.23$ \\
\hline
\end{tabular}


We have investigated the situation with friction coefficient $\mu_{s}$ equal to 0.6 ; the resulting value ranges are listed in Table III. It is found that with $T_{d}$ increasing the range of initial value of $\left(\varphi_{1}, \dot{\varphi}_{1}\right)$ decreases. When $T_{d}$ is equal to 0.14 , the range of $\dot{\varphi}_{10}$ first increases and then decreases as $\varphi_{10}$ increases.

\section{RESULTS AND DISCUSSION}

\subsection{Initial kinematic states}

We choose the unified parameters to generate the leg's motion with friction coefficient $\left(\mu_{s}=0.6\right)$, excitation cycle $\left(T_{d}=0.14\right)$, stance duration $\left(t_{e}=0.2\right)$ and frequency ratio $(s=0.947)$. The resulting dynamics characteristics of varying $\varphi_{10}$ and $\dot{\varphi}_{10}$ are drawn and analysed.
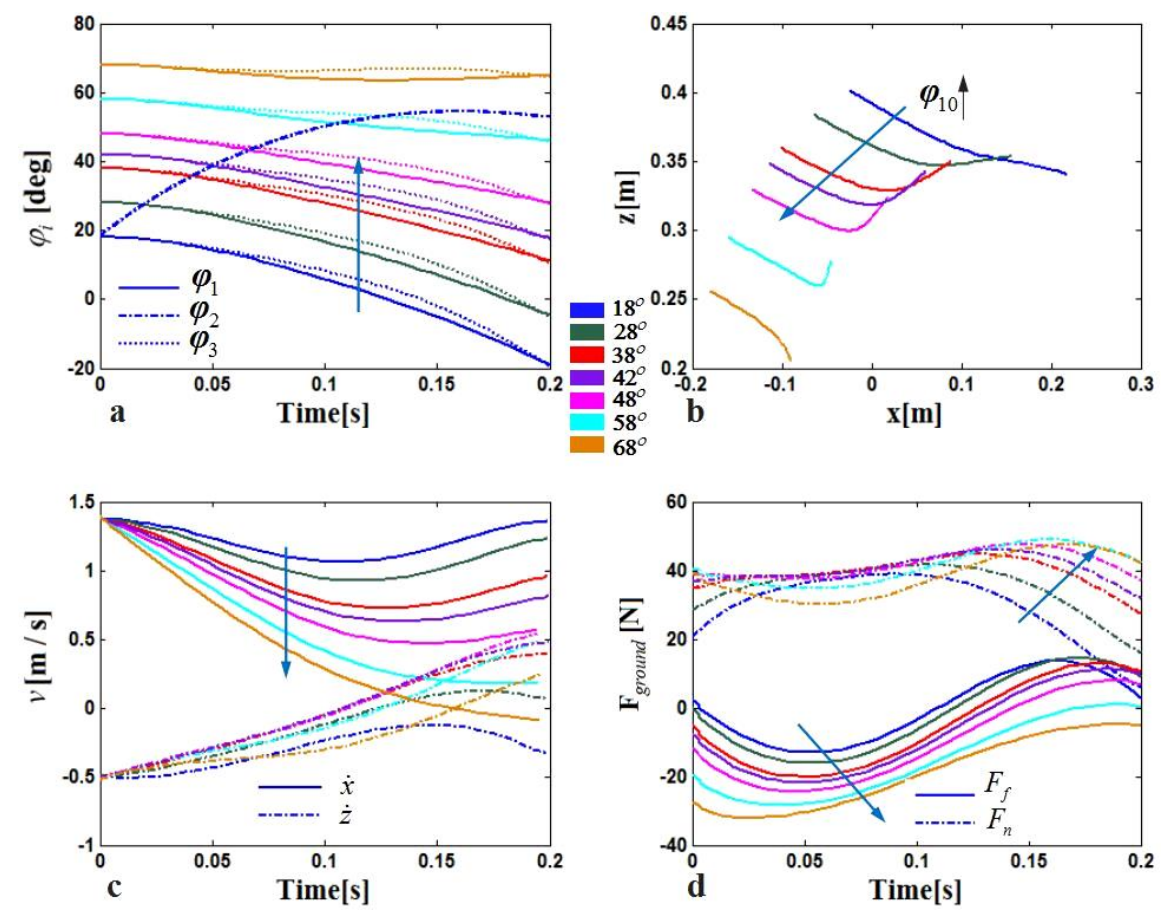

Figure 4: The dynamics characteristics with $\varphi_{10}$ varying.
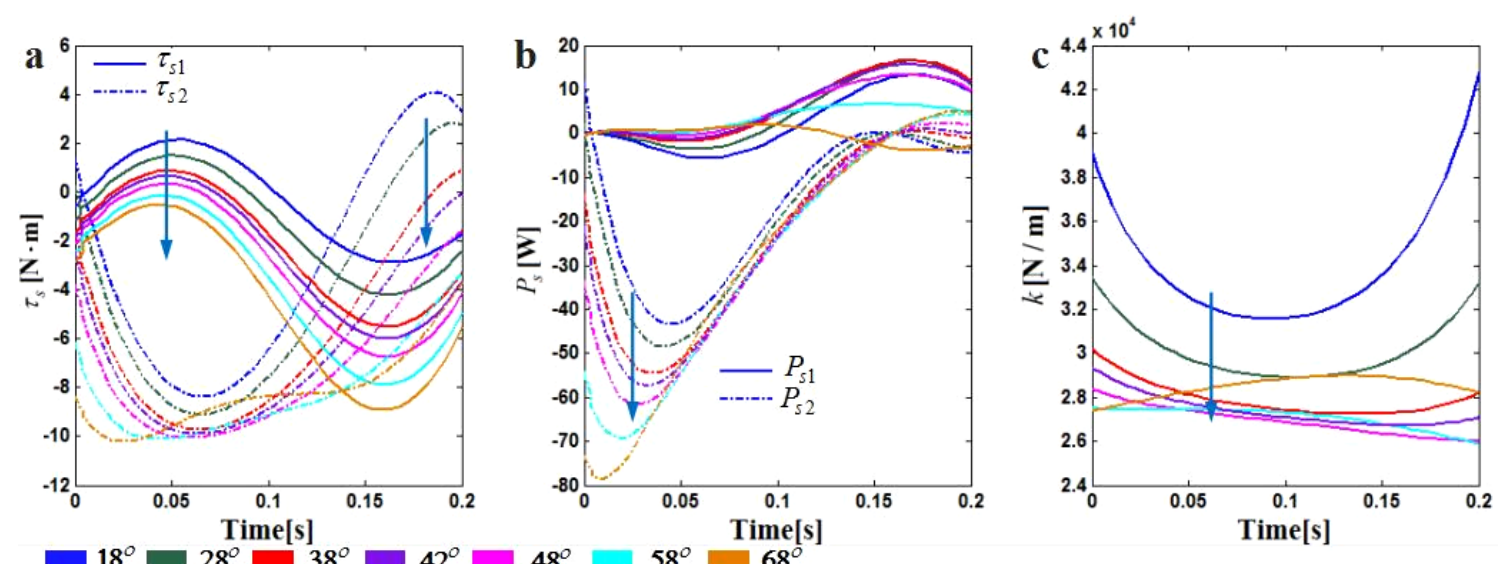

Figure 5: The driven output and variable stiffness with $\varphi_{10}$ varying.

For $\varphi_{10}$, the parameter varies in a range $\left(18^{\circ}, 28^{\circ}, 38^{\circ}, 40^{\circ}, 48^{\circ}, 58^{\circ}, 68^{\circ}\right)$ when $\dot{\varphi}_{10}$ is equal to $0.2 \mathrm{rad} / \mathrm{s}$. To obtain a good knowledge of the resulting motion, the range of $\varphi_{10}$ is extended beyond the stability range in Table III to compare the cases of normal motion and abnormal 
motion. In this situation, the amplitude of generalized elastic deflection $\varphi$ is $3.3^{\circ}$ which is small because of the superposed motion. In Fig. 4 and Fig. 5, different colours refer to dynamics characteristics under different initial condition of $\varphi_{10}$. The arrow indicates the increasing direction of $\varphi_{10}$. Different line types in each subgraph refer to different system parameters.

With $\varphi_{10}$ increasing, the joint trajectory of $\varphi_{1}$ entirely shifts up (see Fig. 4 a). As a result, the trajectory of body mass declines (see Fig. 4 b). Obviously, there is a suitable range in which the motion satisfies the constraint conditions. However, there are different abnormal phenomena with $\varphi_{10}$ less than and more than the suitable initial parameter, as shown in Fig. $4 \mathrm{~b}$. It is found in Fig. $4 \mathrm{c}$ and Fig. $4 \mathrm{~d}$ that if $\varphi_{10}$ is too big, the horizontal velocity will cross zero and the vertical velocity is less than zero all the time. In terms of the ground reaction forces, $F_{f}$ is always in the negative motion direction. Consequently, the body will keep falling and move backward. If $\varphi_{10}$ is too small, the horizontal velocity stays positive, but the vertical velocity is negative all the time as well. In terms of the ground reaction forces, the ratio of $F_{f}$ to $F_{n}$ will be less than the friction coefficient, and $F_{n}$ will be negative at the lift-off event. As a result, the body will fall all the time and even slide and lose the supporting effect of the ground.

It is worth noting that there is a suitable $\varphi_{10}$ to make the stiffness vary linearly. When $\varphi_{10}$ is too high or too small, the changing curve of stiffness is characterized by nonlinearity, as shown in Fig. 5 c. Similarly, the suitable $\varphi_{10}$ corresponds to lower driven torque and power, as shown Fig. 5 a, b.

For $\dot{\varphi}_{10}$, the parameter changes in a range $(-1.2,-0.6,0,0.6,1.2,1.8,2.6)$ (unit: $\mathrm{rad} / \mathrm{s}$ ) when $\varphi_{10}$ is equal to $37^{\circ}$. In this case, there is only one value $(0 \mathrm{rad} / \mathrm{s})$ in the stability range in Table III. Other values are used to investigate abnormal situations. The amplitude of the generalized elastic deflection $\varphi$ is the same as the result of varying $\varphi_{10}$ because of the same generalized vibration parameters. Similar to $\varphi_{10}$, the arrow indicates the increasing direction of $\dot{\varphi}_{10}$.
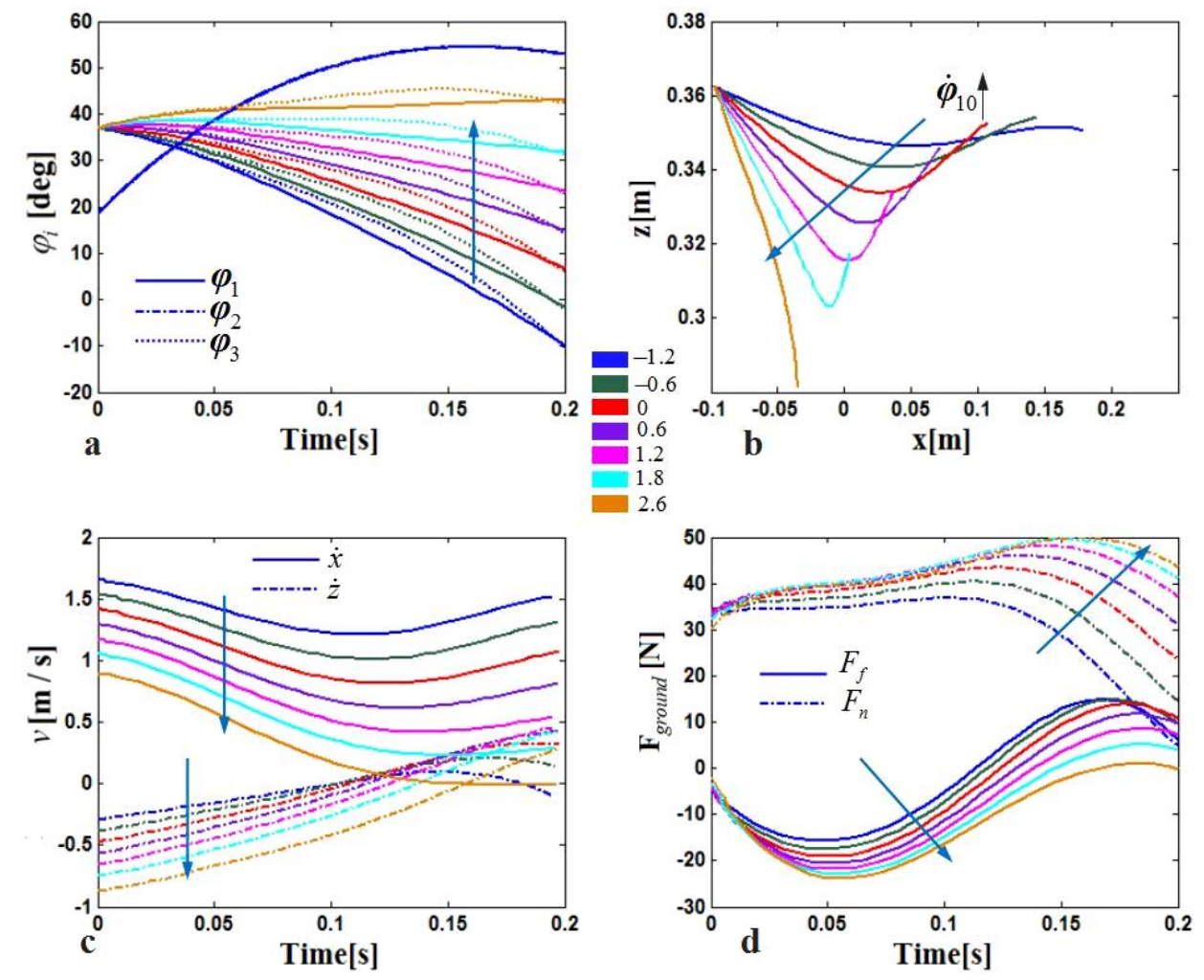

Figure 6: The dynamics characteristics with $\dot{\varphi}_{10}$ varying. 
Similar to varying $\varphi_{10}$, an abnormal motion without meeting the constraint conditions and its cause can be observed in Fig. 6 b. When $\dot{\varphi}_{10}$ is too high, the negative horizontal and vertical velocity and the horizontal ground reaction force will lead to the body falling and moving backward. When $\dot{\varphi}_{10}$ is too low, the negative vertical velocity and lower frictional angle will give rise to the body falling and the foot sliding.
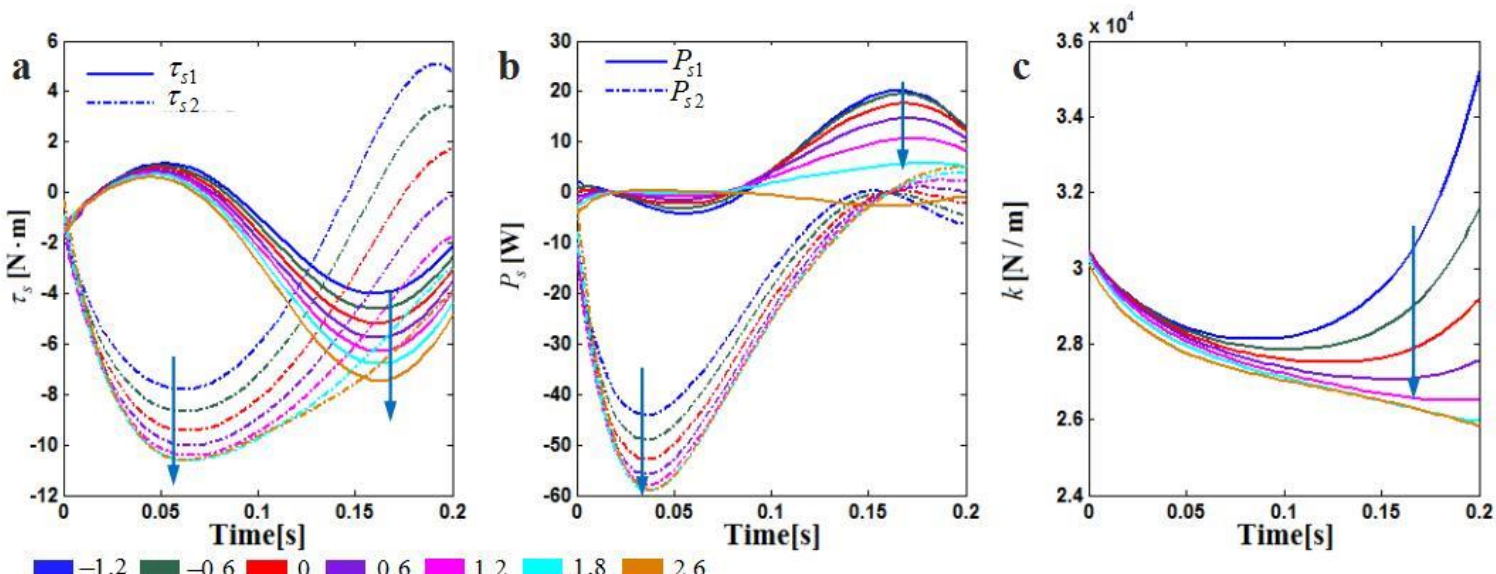

Figure 7: The driven output and variable stiffness with $\dot{\varphi}_{10}$ varying.

On the other hand, the variable stiffness possesses a more linear feature with a higher $\dot{\varphi}_{10}$, as shown in Fig. 7 c. In addition, for driven torque, the higher the value of $\dot{\varphi}_{10}$, the bigger the torque the motors need to output to overcome the dynamics effects of the system, as shown in Fig. 7 a. Furthermore, the higher $\dot{\varphi}_{10}$ results in a higher energy consumption for motor 1, but a lower energy consumption for motor 2 (see Fig. 7 b). Taking all the resulting characteristics into account, a medium value of $\dot{\varphi}_{10}(0 \mathrm{rad} / \mathrm{s})$ is still a suitable initial state to generate stable motion.

\subsection{Dynamics characteristics}

The model generates normal motion primarily by planning the passive motion of the compliant element based on forced vibration and setting suitable initial states. This is a nonlinear and strong coupled problem. Whether motion can be achieved depends entirely on the coupling of dynamic characteristics and generalized vibration characteristics. Therefore, we define the passive motion in advance, which must meet the compression and return process.

Because the ground reaction force in the model is computed based on the centroid theorem, the ground reaction force, joint displacement and angular velocity, body trajectory and moving velocity are correlated. In section 4.1 , it is obviously found in simulation results that the abnormal motion results from the improper moving velocity of the body and is directly caused by improper ground reaction forces. Despite the simplification of the model, it demonstrates that the ground reaction force plays an important role in motion stability, accounting for what role the variable stiffness can play. As shown in Figs. 4 and 6, with a suitable initial kinematic state and variable stiffness, the body moves such as a SLIP moving model. The body pivots about the contact point and the system experiences compression and extension during the stance phase. It is worth noting in the normal situation that starting from the second when the foot of the leg stands on the ground, the horizontal component of the ground reaction force on the leg is negative, and then it changes to positive. The peak vertical force is exerted midway through the stance phase and at this time the horizontal component of force is almost equal to zero. The result is similar to the ground reaction forces of canine 
measured during a single trotting step [16]. Consequently, combining the results of the previous work and this paper, the resulting ground reaction forces meet the biomechanics characteristics and variable stiffness helps to adjust the contact characteristics with the ground.

The dynamics simulation is based on numerical solutions of differential equations; therefore initial kinematic states are very important with respect to the dynamics motion. In the real movement of the leg system, if the system can be input a suitable joint motion to adapt to different moving states, the advantages of the compliant element can be exploited to the greatest extent. It also results in lower driven torque and energy consumption. Furthermore, the motion of $\varphi_{2}$ was planned by the fitting method to be close to a canine's shank motion. It is a fixed input state in this paper. Varying the motion of $\varphi_{2}$ will lead to different states. $\varphi_{2}$ is also an important input that needs to be adjusted to adapt to different moving state.

\subsection{Variable stiffness device}

As observed in section 4.1 , when $\varphi_{10}\left(38^{\circ}, 42^{\circ}\right)$ is in the range $\left(37^{\circ} \sim 47^{\circ}\right)$ of satisfying motion constraint conditions, the needed variable stiffness presents a better linearity. The range of changed stiffness is from $27.24-30.24 \mathrm{KN} / \mathrm{m}$. When $\dot{\varphi}_{10}(0 \mathrm{rad} / \mathrm{s})$ is in the range $(-0.39 \mathrm{rad} / \mathrm{s}$ $\sim 0.06 \mathrm{rad} / \mathrm{s}$ ) of meeting motion constraint conditions, although the linearity of the needed variable stiffness in this situation is not the best, it imposes a relatively smaller stiffness range 27.47-30.65 KN/m.

If the variable stiffness device has a good adjustability feature and a large adjustable range, it will be applied to quadruped robots by suitable design, and the robots will be able to adapt to a large range of stable motion. Compared with the variable stiffness methods of spring preload in the reference [17], the structure-controlled stiffness is able to qualify for the situation, because it adjusts stiffness by changing active length and the stiffness is an independent parameter, such as the Tunable Stiffness Mechanism (TSM) [18]. The TSM consists of an inner spring, an outer spring and a piece, and they are arranged in the series and parallel configurations. The stiffness of TSM is varied by rotating the inner piece to change different series and parallel configurations. Because of TSM's good adjustability feature, through compact designing for the variable stiffness device, including the transmission to rotate the piece and a suitable control system, it can be applied to legged motion. The only downside is that there is friction when the piece is rotated.

\section{CONCLUSIONS}

This paper focuses on an investigation of the motion of a specific leg model during the stance phase based on dynamics analyses. Based on dynamics equations that have a similar structure to the formula for forced vibration, the motion is planned and created under certain constraint conditions. Furthermore, the effects of generalized vibration parameters on the motion creation are evaluated to obtain the optimum combination of these parameters. In the simulation experiment, the initial motion states are varied to study the dynamics characteristics of the leg model and its sensitivity to variable stiffness. It is found that the normal motion captures a property of ground reaction forces similar to mammal runners' motion. In addition, the required stiffness to create motion, as well as the range of stiffness variation, suggest that it can be achieved by a suitable variable stiffness actuator and transmission mechanism.

A natural continuation of our work is to extend the presented study to a complete cycle, to investigate the effects of variable stiffness on generating a periodic motion. Because of the existence of friction in adjusting stiffness, the damping element will also be taken into 
account in the leg model in the future. The corresponding planning and control method should be studied and a single-leg motion prototype needs to be established.

\section{ACKNOWLEDGEMENT}

An earlier version of this paper was presented at the 2017 ASME Dynamic System and Control Conference. The research work was supported by the Natural Science Fundament of China under Grant No. 51575409 and the Fundamental Research Funds for the Central Universities (WUT: 173204001 and 175204003).

\section{REFERENCES}

[1] Alexander, R. M. (1990). Three uses for springs in legged locomotion, The International Journal of Robotics Research, Vol. 9, No. 2, 53-61, doi:10.1177/027836499000900205

[2] Dickinson, M. H.; Farley, C. T.; Full, R. J.; Koehl, M. A. R.; Kram, R.; Lehman, S. (2000). How animals move: an integrative view, Science, Vol. 288, No. 5463, 100-106, doi:10.1126/ science.288.5463.100

[3] Cavagna, G. A.; Saibene, F. P.; Margaria, R. (1964). Mechanical work in running, Journal of Applied Physiology, Vol. 19, No. 2, 249-256

[4] Zhou, X.; Bi, S. (2012). A survey of bio-inspired compliant legged robot designs, Bioinspiration \& Biomimetics, Vol. 7, No. 4, Paper 041001, doi:10.1088/1748-3182/7/4/041001

[5] Ferris, D. P.; Louie, M.; Farley, C. T. (1998). Running in the real world: adjusting leg stiffness for different surfaces, Proceedings of the Royal Society B: Biological Sciences, Vol. 265, No. 1400, 989-994, doi:10.1098/rspb.1998.0388

[6] Vanderborght, B.; Van Ham, R.; Lefeber, D.; Sugar, T. G.; Hollander, K. W. (2009). Comparison of mechanical design and energy consumption of adaptable, passive-compliant actuators, The International Journal of Robotics Research, Vol. 28, No. 1, 90-103, doi:10.1177/ $\underline{0278364908095333}$

[7] Blickhan, R. (1989). The spring mass model for running and hopping, Journal of Biomechanics, Vol. 22, No. 11-12, 1217-1227, doi:10.1016/0021-9290(89)90224-8

[8] Poulakakis, I.; Smith, J. A.; Buehler, M. (2005). Modeling and experiments of untethered quadrupedal running with a bounding gait: the Scout II robot, International Journal of Robotics Research, Vol. 24, No. 4, 239-256, doi:10.1177/0278364904050917

[9] Gan, Z.; Wiestner, T.; Weishaupt, M. A.; Waldern, N. M.; Remy, C. D. (2015). Passive dynamics explain quadrupedal walking, trotting, and tolting, Journal of Computational and Nonlinear Dynamics, Vol. 11, No. 2, Paper 021008, 12 pages, doi:10.1115/1.4030622

[10] Deban, S. M.; Schilling, N.; Carrier, D. R. (2012). Activity of extrinsic limb muscles in dogs at walk, trot and gallop, Journal of Experimental Biology, Vol. 215, No. 2, 287-300, doi: $10.1242 /$ jeb.063230

[11] Sudharsan, J.; Karunamoorthy, L. (2016). Path planning and co-simulation control of 8 DOF anthropomorphic robotic arm, International Journal of Simulation Modelling, Vol. 15, No. 2, 302-312, doi:10.2507/IJSIMM15(2)9.339

[12] Li, Z.; Tan, Y. G.; Hong, L.; Dhupia, J. S.; Zeng, S.; Huang, L. K. (2017). A single leg model with a novel variable stiffness element based on the dynamics analysis and its dynamic characteristics, Proceedings of the ASME 2017 Dynamic Systems and Control Conference, 8 pages, doi:10.1115/DSCC2017-5057

[13] Seok, S.; Wang, A.; Chuah, M. Y. M.; Hyun, D. J.; Lee, J.; Otten, D. M.; Lang, J. H.; Kim, S. (2015). Design principles for energy-efficient legged locomotion and implementation on the MIT Cheetah robot, IEEE/ASME Transactions on Mechatronics, Vol. 20, No. 3, 1117-1129, doi:10.1109/TMECH.2014.2339013

[14] Nie, H.; Sun, R.; Guo, C.; Qin, G.; Yu, H. (2015). Innovative design and performance evaluation of a high-speed bionic mechanical leg, Journal of Bionic Engineering, Vol. 12, No. 3, 352-360, doi: $10.1016 / \mathrm{S} 1672-6529(14) 60127-X$ 
[15] Sato, R.; Miyamoto, I.; Sato, K., Ming, A.; Shimojo, M. (2015). Development of robot legs inspired by bi-articular muscle-tendon complex of cats, 2015 IEEE/RSJ International Conference on Intelligent Robots and Systems, 1552-1557, doi:10.1109/IROS.2015.7353574

[16] Lee, D. V.; Bertram, J. E. A.; Todhunter, R. J.; (1999). Acceleration and balance in trotting dogs, Journal of Experimental Biology, Vol. 202, No. 24, 3565-3573

[17] Vanderborght, B.; Albu-Schaeffer, A.; Bicchi, A.; Burdet, E.; Caldwell, D. G.; Carloni, R.; Catalano, M.; Eiberger, O.; Friedl, W.; Ganesh, G.; Garabini, M.; Grebenstein, M.; Grioli, G.; Haddadin, S.; Hoppner, H.; Jafari, A.; Laffranchi, M.; Lefeber, D.; Petit, F.; Stramigioli, S.; Tsagarakis, N.; Van Damme, M.; Van Ham, R.; Visser, L. C.; Wolf, S. (2013). Variable impedance actuators: a review, Robotics and Autonomous Systems, Vol. 61, No. 12, 1601-1614, doi:10.1016/j.robot.2013.06.009

[18] Qaiser, Z.; Kang, L.; Johnson, S. (2017). Design of a bioinspired tunable stiffness robotic foot, Mechanism and Machine Theory, Vol. 110, 1-15, doi:10.1016/j.mechmachtheory.2016.12.003 